\title{
DaVinci Canvas: \\ A Telerobotic Surgical System with Integrated, Robot-Assisted, Laparoscopic Ultrasound Capability
}

\author{
Joshua Leven ${ }^{1}$, Darius Burschka ${ }^{1}$, Rajesh Kumar $^{2}$, Gary Zhang ${ }^{2}$, \\ Steve Blumenkranz ${ }^{2}$, Xiangtian (Donald) Dai ${ }^{1}$, Mike Awad ${ }^{1}$, Gregory D. Hager ${ }^{1}$, \\ Mike Marohn ${ }^{1}$, Mike Choti ${ }^{1}$, Chris Hasser ${ }^{2}$, and Russell H. Taylor ${ }^{1}$ \\ ${ }^{1}$ The Johns Hopkins University, Baltimore, Maryland 21218 USA \\ rhtejhu.edu \\ ${ }^{2}$ Intuitive Surgical, Inc., Sunnyvale, CA 94086 \\ Chris.Hasser@intusurg.com
}

\begin{abstract}
We present daVinci Canvas: a telerobotic surgical system with integrated robot-assisted laparoscopic ultrasound capability. DaVinci Canvas consists of the integration of a rigid laparoscopic ultrasound probe with the daVinci robot, video tracking of ultrasound probe motions, endoscope and ultrasound calibration and registration, autonomous robot motions, and the display of registered 2D and 3D ultrasound images. Although we used laparoscopic liver cancer surgery as a focusing application, our broader aim was the development of a versatile system that would be useful for many procedures.
\end{abstract}

\section{Introduction}

Open surgery serves as the gold standard treatment for many disorders, despite the increased morbidity associated with laparotomy. Minimally invasive techniques have developed to reduce this morbidity and improve the quality of life following surgery. In exchange for this lower morbidity, clinicians and patients have, at times, accepted lower efficacy, and surgeons have tolerated more difficult working conditions. The daVinci ${ }^{\circledR}$ surgical system gives surgeons dexterous laparoscopic tools, intuitive motion, and a high-fidelity $3 \mathrm{D}$ vision system, allowing them to achieve outcomes comparable or better to that of open surgery, with the low morbidity of minimally invasive surgery (e.g., [1]). However, the "classical telesurgery" approach of the daVinci essentially is limited to replicating 20th century open surgery in an MIS environment.

Primary liver cancer is one of the most common malignancies, accounting for more than 1 million cases per year [2]. Hepatic metastases, or secondary liver cancer, is also a common disease facing the clinician. IOUS-directed procedures such as liver biopsy, tumor ablation, and hepatic resection today often require trade-offs between efficacy and morbidity. While liver resection is most often performed using open laparotomy, percutaneous approaches are often used to perform needle biopsy or ablation. This technique has the advantage of potentially lower morbidity, at least compared to open surgery, and it can employ various imaging modalities, including US, CT, and MRI. However, there are several significant advantages to the performance of ablation during laparotomy or laparoscopy. First, surgical procedures 
provide enhanced staging, as laparotomy and laparoscopy afford the opportunity to identify both hepatic and extra-hepatic metastases not visualized on preoperative imaging. Intraoperative ultrasonography (IOUS) capitalizes on the ability to place the ultrasound probe directly on the liver surface. Second, laparotomy or laparoscopy affords improved access to tumors in difficult locations. Third, in cases of multiple tumors, biopsy and ablation can be combined with resection when using an operative approach. Finally, clinical studies demonstrate that open surgical ablation likely has improved outcomes compared to percutaneous approaches [3].

IOUS of the liver [4] is widely used for staging, ablation, and planning for resection. It is the most accurate method for detecting liver metastases, with accuracy rates above 90 percent [27]. Despite theoretical advantages, intraoperative laparoscopic ultrasound (LUS) is not widely practiced. In situations in which LUS has been utilized, the results are not comparable to that of open operative ultrasonography. Limitations to this technique among general and hepatobiliary surgeons relate to the its technical difficulty, to the imprecise methods of manual freehand probe manipulation, limited degree of positioning, and 2D imaging [5] .

This paper reports our initial steps in integrating robot-assisted laparoscopic ultrasound (RLUS) into the daVinci surgical robot system. Our general goal is to enable surgeons to perform minimally invasive liver interventions with the efficacy of open surgery. Although we have used liver surgery as a focusing application, our broader aim is to develop a generally useful LUS capability for many surgical fields, including general, cardiac, gynecologic, and urologic surgery.

\section{Background}

Several groups have active programs in robotically-assisted ultrasonography. Fenster, et al. have a long record of using tracked and robotically-manipulated 2D US probes to produce 3D US images, as well as recent work on robotically-assisted TRUSguided prostate brachytherapy [6]. Several groups have reported systems using ultrasound-based targeting to assist in other robotically-assisted percutaneous procedures [7,8] and other groups (e.g., [9-12]) have developed roboticallymanipulated extracorporeal ultrasound systems. None of these systems involve LUS or integrate US into an interventional procedure. There has been one experimental system for remote LUS probe manipulation [13] as part of a 1998 EU telemedicine initiative. We have not been able to find any subsequent publication of this work.

Many groups have explored the use of navigational tracking devices with transcutaneous 2D and 3D ultrasound, including work targeted at the liver (e.g., $[14,15])$ There have been recent efforts to apply navigation techniques to LUS (e.g., [16-18]) and work on "augmented reality" for US using head mounted displays [19]. There has also been a body of work on human-machine cooperative systems implementing "virtual fixtures" or the equivalent for telesurgery (e.g., [20-22]).

\section{System Overview}

A block diagram of our system appears in Fig 1-A. It consists of a standard daVinci surgical robot equipped with a special LUS tool (Fig 1-B,C), consisting of a $10 \mathrm{~mm}$ 


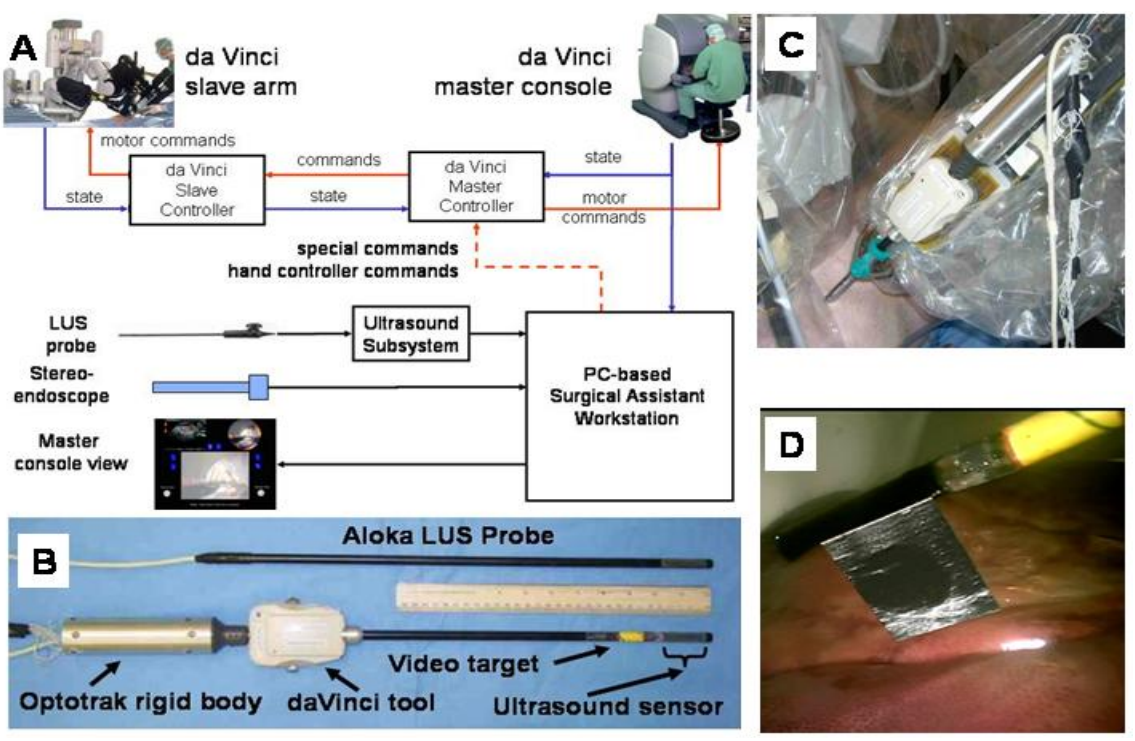

Fig. 1. (A) Block diagram; (B) LUS tool with Optotrak tracking body; (C) LUS tool held by daVinci in pig cadaver; (D) view through surgeon console of LUS probe with video tracking target and video overlay of LUS image

diameter Aloka LUS probe held by a specially constructed daVinci interface unit that is capable of rotating the LUS probe about its axis. We have added a distinctive pattern to the shaft of the probe near the LUS sensor (Fig 2-A) that can easily be located by computer vision techniques. The back end of the LUS probe is equipped with a specially constructed Optotrak ${ }^{\circledR}$ tracking body for use in system calibration and validation experiments. To this, we added a PC-based "surgical assistant" workstation which communicated with the daVinci master console through serial links and research application programming interface (API) provided under special agreement by Intuitive Surgical, Inc. Both video channels from the daVinci's stereoendoscope were fed through video frame grabber cards attached to the workstation, permitting us to perform real time image processing. LUS images were captured from the Aloka video console through a third frame grabber. State information from the daVinci was

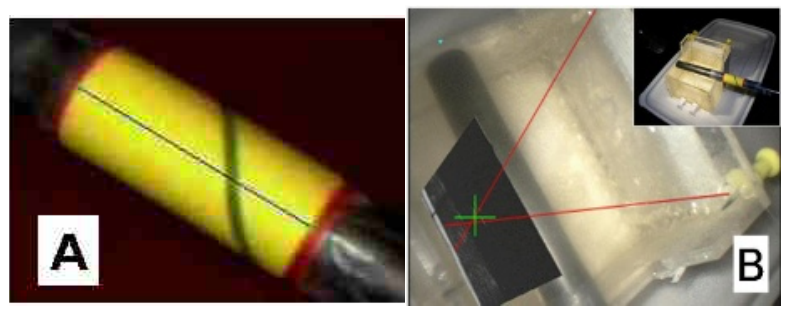

Fig. 2. (A) Example of the ultrasound probe with tracking marker and detected shaft axis (blue line -- graphic overlay). (B) Overlay Accuracy Phantom being used with the LUS probe. (Inset) Probe sitting in the phantom. (Main image) The extrapolated location of the intersection point in camera view (red), and the ultrasound crosswire segmentation (green). 
transmitted to the workstation through Ethernet and a limited repertoire of motion commands were sent to the daVinci through a serial interface.

Video tool tracking: To demonstrate the feasibility of visual tool tracking, the ultrasound probe was marked with a yellow sticker with green and red stripes as shown in Fig 2-A, and a segmentation algorithm located the center of the sticker in 2D image space. The yellow sticker helped locate the shaft centerline, the green spiral line helped compute the twist angle of the shaft while the red stripes helped the extraction of the end points of this centerline.

Once the centerlines are detected in both stereo cameras, we compute the $3 \mathrm{D}$ axis of the shaft from the intersection of two planes formed by the shaft centerline and the optical origin in the left and right cameras respectively.

Calibration and registration: DaVinci Canvas requires several calibration and registration steps, all implemented by leveraging prior work in our laboratory.

Laparoscopic Ultrasound Calibration - Ultrasound image coordinates were calibrated to the Optotrak rigid body using an $\mathrm{AX}=\mathrm{XB}$ formulation [23]. The ultrasound probe was placed in three known orientations in a specially constructed calibration phantom \{Viswanathan, $2004 \# 1599$ \}. The three poses allow three relative transformations based on Optotrak readings (A) and three relative transformations based on the ultrasound images $(\mathrm{B})$ for the $\mathrm{AX}=\mathrm{XB}$ registration.

Stereo Endoscope Calibration and Registration - In order to determine the intrinsic and extrinsic parameters of the stereo endoscope, we used our checkerboard phantom with the multi-plane formulation provided by the Caltech Image Calibration Toolkit. We added Optotrak markers to a typical checkerboard video calibration phantom and digitized each corner of the checkerboard using a calibrated Optotrak pointer. These corner coordinates were then used with the camera calibration to perform a pointcloud to point-cloud registration between the endoscope rigid body and camera frame.

DaVinci Robot API Registration - The daVinci robot API uses the robot kinematics to report a coordinate frame for the endoscope and ultrasound tool tip. However, due to inaccuracies in the setup joint encoders, both of these coordinate frames were offset from their correct values. Thus, it was necessary to register the offsets between the real camera frame and the camera frame calculated from the kinematics as well as between the real and kinematic ultrasound probe frames. Then, the kinematics could be used in place of the Optotrak readings to determine ultrasound image overlay placement.

We assumed a constant transformation between the kinematic tool tip and the LUS probe Optotrak rigid body. This is reasonable so long as the position of the camera does not overly change. Using an $\mathrm{AX}=\mathrm{XB}$ formulation, the probe was moved to several positions, and the
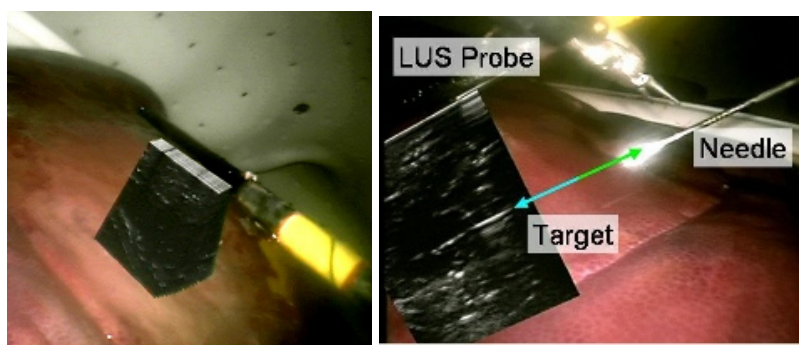

Fig. 3: (left) 3D LUS image obtained by "rocking"; (right) 2D LUS of lesion during targeting task (graphics added manually) 
static offset between tool tip and rigid body was registered. Knowing this offset, the camera offset could be calculated directly.

Ultrasound Image Display: DaVinci Canvas includes the ability for the user to view the ultrasound image as a picture-in-picture (PIP) insert, or as an overlay that appears to fan out from the ultrasound transducer itself, providing a visualization similar to the "ultrasound flashlight" concept of Stetten et al. [25](Fig 1-D). Our system includes a simple 3D ultrasound overlay volume rendering implementation based on [26] (Fig. 3 - Left), and was evaluated by performing a needle insertion task.

Autonomous Motions: Robotic ultrasound has the potential to reduce variability in the images produced, compared to freehand scanning, and can reduce operator workload and difficulty. Behaviors as simple as rocking the probe back and forth can maintain an updated 3D image without operator intervention. In daVinci Canvas, surgeons can rock the probe to one extreme, squeeze the daVinci grip, rock to the other extreme, and release the grip to easily specify automated rocking. Surgeons could also use this same click and drag paradigm to initiate repeating trajectories in Cartesian space.

\section{System Accuracy}

We evaluated the accuracy of the system under various calibration conditions and determined an error of $2.83 \pm 0.83 \mathrm{~mm}$ using Optotrak exclusively, and $2.16 \pm 1.43$ $\mathrm{mm}$ using registered daVinci kinematics. We defined accuracy error in this case as the 3D displacement between where a feature appears in the ultrasound image overlay, and where that same feature appears in the endoscope video.

Accuracy assessment was performed using our overlay accuracy phantom (Fig 2B). The phantom is essentially a crosswire calibration phantom with a significant fraction of the two wires visible above the water line. The crosswire point was localized using the ultrasound overlay and compared to the intersection point of the two wires visible above the water. The extracted points were used to determine two 3D positions in the camera frame - one due to the video, and one due to the overlay. We considered the distance between these two points to be the accuracy error.

\section{Surgeon Evaluation}

The clinical evaluation team included two attending general surgeons with significant robotic surgery and ultrasound experience, three surgery residents, and a physician with extensive robotic surgery and customer interface experience. The clinical evaluation team assessed ease of use, reliability, clinical strengths, and limitations of the robot assisted laparoscopic ultrasound probe.

Staging Task (Lesion Finding): Our objective in the staging task was to compare the efficacy of freehand laparoscopic ultrasound with robotic laparoscopic ultrasound in locating simulated liver lesions. Lesions were created using $2 \%$ agarose in water, and placed in ex-vivo porcine liver. Four livers were used for the staging experiment. The number, size, and location of lesions within each liver were randomized. Six surgeons 
found the lesions with freehand LUS, daVinci LUS without automated motions, and daVinci LUS with automated motions.

Results: As expected during freehand ultrasound, advanced surgeons performed better than intermediate and novice surgeons in nearly all categories studied. Likewise, intermediates performed better than novices in the number of lesions correctly identified and identifying correctly the location and size of lesions. Best performance was observed from advanced surgeons using freehand ultrasound. Advanced, intermediate, and novice surgeons performed similarly when using robotic assistance. The longest time to identify each lesion was observed when using robotic assistance with automation.

Discussion: While the robotic techniques did not perform at the level of advanced surgeons using the freehand technique, the robot served as the "equalizer," allowing novices to perform at the level of more experienced surgical ultrasonographers or better. The rigidity of the ultrasound probe significantly limited motion within the simulated abdomen. Almost all surgeons felt it would be important to have a flexible ultrasound probe that would return degrees of freedom characteristic of the daVinci end effectors. Another important feature universally desired by surgeons was a marking tool to determine if it was a new lesion or one previously encountered. Clinically, this was felt to be important in reducing the number of missed lesions.

Of all the robotic ultrasound features available to the surgeon, overlay and PIP were found to be the most useful. These allowed rapid identification and subsequent characterization of lesions. Automation features were less useful, although these features may be useful with daVinci models having 3 instrument manipulation arms in addition to the endoscope arm. In this case, the surgeon could use two arms to perform the procedure while the third instrument holds the LUS probe and initiates "rocking" to obtain 3D images or 2D sweeps over a lesion or critical structure.

This pilot experiment served as a learning exercise, with a rough prototype and small sample size not intended to show quantitative superiority of robotic ultrasound over freehand ultrasound. The key result was qualitative: Surgeons felt that with an articulated probe and the ability to annotate lesions, the system would have a competitive advantage over freehand ultrasound, particularly when used as an aid to needle targeting or resection tasks. They also felt that it would lower the barriers to the use of laparoscopic ultrasound by making it easier to learn.

Lesion Targeting Task: We performed some preliminary investigation into the use of registered ultrasound overlays in guiding needles to target lesions. Livers were prepared as in the staging task. A laparoscopic surgeon skilled in ultrasound techniques performed the targeting task using either freehand ultrasound and needle or using daVinci assistance with a long rigid needle in the left grasper and the ultrasound probe in the right arm. The long needle was passed through the simulated torso and its pivot point was fixed at the "skin." Three targeting tasks were performed, one laparoscopic freehand (long needle only) and twice robotically.

Results and Discussion: Freehand placement accuracy was $5.4 \mathrm{~mm}$ and robot-assisted accuracy was comparable $(4.5 \mathrm{~mm} \& 7.5 \mathrm{~mm})$. For this anecdotal $(\mathrm{N}=1)$ experiment, the surgeon comments were more informative. The surgeon suggested it would be helpful to have a flexible daVinci ultrasound probe, and an extrapolation of the needle trajectory displayed in the daVinci console. 


\section{Conclusions and Future Work}

This work was phase one of a larger effort directed toward a complete integrated robotic system for LUS-assisted hepatic surgery and (more generally) toward development of a new generation of information rich surgical assistant systems. Our initial results are very encouraging. The ability of the ultrasound dynamic image to be displayed in 'real time' at the point of scanning is a powerful tool, enhancing ease of use and surgeon situational awareness.

Clinically acceptable accuracies can be achieved using an Optotrak or directly using daVinci kinematics. The rigid ultrasound probe (versus flexible) was a limitation in scanning selected sectors of the operative field. We had hoped that a volume display would improve the speed and accuracy of such a procedure. However, in its current state, it was found to be less helpful and even distracting when compared to the $2 \mathrm{D}$ ultrasound overlay. The registered $2 \mathrm{D}$ overlay exceeded our expectations by proving capable of assisting needle targeting procedures.

Future plans include development of more robust, capable tools \& image processing algorithms, further evaluation in vivo, and eventual clinical development

\section{Acknowledgments}

At the risk of some injustice to the many colleagues who assisted in various ways, we would like to extend special thanks to Emad Boctor for ultrasound expertise; to Iulian Iorchidata for help with test apparatus; and to Randy Brown and Sue Eller of JHU's MIS Training Center for help with JHU animal trials. This work was funded in part by NIH grant R41 RR019159, in part by the NSF under agreement EEC9731478, and in part by JHU internal funds. We thank Aloka for the loan of LUS equipment.

\section{References}

1. T. Ahlering, D. Woo, E. L, and others, "Robot-assisted versus open radical prostatectomy: a comparison of one surgeon's outcomes.," Urology, vol. 63, pp. 819-822, 2004.

2. E. Nakakura and M. Choti, "Hepatocellular Carcinoma: Current Management Recommendations," Advances on Onc, vol. 16(2), pp. 12-18, 2000.

3. T. Wood and others, "Radiofrequency ablation of 231 unre-sectable hepatic tumors: indications, limitations, and complications," Ann. Surg. Oncol., vol. 7, pp. 593-600, 2000.

4. R. A. Kane, "Intraoperative Ultrasonography, History, Current State of the Art, and Future Directions," J Ultrasound Med, vol. 23, pp. 1407-1420, 2004.

5. B. Rau, M. Hunerbein, and P. Schlag, "Is There Additional Information From Laparoscopic Ultrasound in Tumor Staging?," Dig Surg, vol. 19(6), pp. 479-83, 2002.

6. Z. Wei, G. Wan, L. Gardi, and others, "Robot-assisted 3D-TRUS guided prostate brachytherapy: system integration and validation," Medical Physics, vol. 31(3), pp. 539548, 2004.

7. J. Stoll, P. Dupont, and R. Howe, "Ultrasound-based servoing of manipulators for telesurgery," in SPIE Telemanipulator and Telepresence Technologies VIII, vol. 4570. Newton, Mass.: SPIE, 2001.

8. E. Boctor and others, "Dual-Armed Robotic System for Intraoperative Ultrasound Guided Hepatic Ablative Therapy: A Prospective Study," in IEEE 2004 International Conference on Robotics and Automation. New Orleans,LA, 2004, pp. 2517-2522. 
9. E. Degoulange, L. Urbain, P. Caron, S. Boudet, J. Gariepy, L. Megnien, F. Perrot, and E. Dombre, "HIPPOCRATE: an intrinsically safe robot for medical applicaions," in IEE/RSH International Conference on Intelligent Robots and Systems. Victoria, 1998, pp. 959-964.

10. P. Abolmaesumi, S. E. Salcudean, W. H. Zhu, M. R. Sirouspour, and S. P. DiMaio, "Image-Guided Control of a Robot for Medical Ultrasound," IEEE Transactions on Robotics and Automation, vol. 18(1), pp. 11-23, 2002.

11. M. Mitsuishi, S. i. Warisawa, T. Tsuda, T. Higuchi, N. Koizumi, H. Hashizume, and K. Fujiwara, "Remote Ultrasound Diagnostic System," in Proc. IEEE Conf. on Robotics and Automation. Seoul, 2001, pp. 1567-1574.

12. A. Vilchis, J. Troccaz, P. Cinquin, K. Masuda, and F. Pellissier, "A New Robot Architecture for Tele-Echography," IEEE Trans. Robotics \& Automation, vol. 19(5), pp. 922-926.

13. D. d. Cunha, P. Gravez, C. Leroy, E. Maillard, J. Jouan, P.Varley, M. Jones, M. Halliwell, D. Hawkes, P. N. T. Wells, and L. Angelini, "The MIDSTEP System for Ultrasound guided Remote Telesurgery .," in IEEE EMBS, vol. 20/3: IEEE, 1998, pp. 1266-1269.

14. J. Stefansic, A. Herline, Y. Shyr, W. Chapman, J. Fitzpatrick, B. Dawant, and R. J. Galloway, "Registration of Physical Space to Laparoscopic image space for use in minimally invasive hepatic surgery," IEEE Trans Med Imaging, vol. 19(10), pp. 1012-1023, 2000.

15. T. Lange, S. Eulenstein, M. Hunerbein, H. Lamecker, and P.-M. Schlag, "Augmenting Intraoperative 3D Ultrasound with Preoperative Models for Navigation in Liver Surgery," in MICCAI 2004, vol. LNCS 3217. St. Malo: Springer, 2004, pp. 534-541.

16. D. Wilheim, H. Feussner, A. Schneider, and J. Harms, "Electromagnetically navigated laparoscopic ultrasound," Surg. Technol. Int., vol. 11, pp. 50-54, 2003.

17. J. Ellsmere, J. Stoll, D. Rattner, and D. Brooks, "A navigation system for augmenting laparoscopic ultrasound," in Medical Image Computing and Computer-Assisted Intervention - MICCAI 2003: Springer, 2003, pp. 184-191.

18. P. M. Novotny, J. W. Cannon, and R. D. Howe, "Tool Localization in 3D Ultrasound Images," in MICCAI 2003, vol. LNCS 2879. Montreal: Springer, 2003, pp. 969-970.

19. H. Fuchs, M. A. Livingston, R. Raskar, D. n. Colucci, K. Keller, A. State, J. R. Crawford, P. Rademacher, S. H. Drake, and A. A. Meyer, "Augmented Reality Visualization for Laparoscopic Surgery," in MICCAI '98. Boston: Springer, 1998.

20. B. Davies, S. Harris, M. Jakopec, and J. Cobb, "A novel hands-on robot for knee replacement surgery," in Computer Assisted Orthopaedic Surgery USA (CAOS USA), A. DiGioia and B. Jaramaz, Eds. Pittsburgh: UPMC Medical Center, 1999, pp. 70-74.

21. A. Bettini, S. Lang, A. Okamura, and G. Hager, "Vision Assisted Control for Manipulation Using Virtual Fixtures: Experiments at Macro and Micro Scales," IEEE International Conference on Robotics and Automation, 2002.

22. M. Li and R. H. Taylor, "Performance of Teleoperated and cooperatively controlled surgical robots with automatically generated spatial virtual fixtures.," in IEEE International Conference on Robotics and Automation, 2005, pp. accepted.

23. E. Boctor, A. Viswanathan, M. Choti, R. Taylor, G. Fichtinger, and G. Hager, "A Novel Closed Form Solution For Ultrasound Calibration," in International Symposium on Biomedical Imaging. Arlington, VA, 2004, pp. 527-530.

24. A. Viswanathan, E. M. Boctor, R. H. Taylor, G. D. Hager, and G. Fichtinger, "Immediate Ultrasound Calibration with Three Poses and Minimal Image Processing," in MICCAI, vol. 2, C. B. D. R. H. P. Hellier, Ed. Saint-Malo, France, 2004, pp. 446-454.

25. G. Stetten and V. Chib, "Overlaying Ultrasound Images on Direct Vision," Journal of Ultrasound in Medicine, vol. 20(3), pp. 235-240, 2001.

26. W. F. Garrett, H. Fuchs, M. C. Whitton, and A. State, "Real-Time Incremental Visualization of Dynamic Ultrasound Volumes Using Parallel BSP Trees," in Visualization '96, 1996.

27. J. Zacherl, C. Scheuba, M. Imhof, M. Zacherl, F. Langle, P. Pokieser, F. Wrba, E. Wenzl, F. Muhlbacher, R. Jakesz, R. Steininger. Current value of intraoperative sonography during surgery for hepatic neoplasms. World J Surg. 2002 May;26(5):550-4. 